\title{
Metastasis to the parotid region as an initial presentation of renal cell carcinoma: A case report
}

\author{
CHAI YANLAN, SONG LIPING, CHE SHAOMIN and LIU ZI \\ Cancer Center, First Hospital of Xi'an Jiaotong University, Shaan'xi, Xi'an 710061, P.R. China
}

Received September 23, 2012; Accepted December 10, 2012

DOI: $10.3892 / \mathrm{ol} .2013 .1110$

\begin{abstract}
Distant metastasis of renal cell carcinoma (RCC) to the parotid region is extremely rare, particularly as an initial presentation. In the present study, we report a rare case of parotid region metastasis from RCC as an initial presentation in a 44-year-old female who presented with a painless lump in the right parotid region. Investigation revealed RCC in the left renal region and metastasis to the right iliac area. A radical nephrectomy was performed but the patient refused any further treatment. After seven months, the patient reappeared with systemic multiple metastases, with the exception of previous metastases that were enlarging significantly. On admission, interleukin-2 and local radiotherapy were administered. However, oral mucositis occurred. Targeted therapy with sunitinib was recommended.
\end{abstract}

\section{Introduction}

Metastases of malignancies to the parotid region are relatively infrequent (21-42\% of all malignant tumors) and originate primarily from head and neck squamous cell carcinoma and melanoma of the skin (1). Metastases of an infraclavicular origin are rare $(0.16-4 \%)(1,2)$.

Renal cell carcinoma (RCC) is known for its high propensity for early metastasis and $\leq 1 / 3$ of patients diagnosed with RCC present with metastatic disease at the time of diagnosis (3). Although RCC may metastasize to any organ system, its metastasis to the maxillofacial area is a relatively rare phenomenon. This study describes a patient whose first clinical presentation of RCC was parotid region metastasis. To

Correspondence to: Professor Song Liping, Cancer Center, First Hospital of Xi'an Jiaotong University, 277 Yanta West Road, Shaan'xi, Xi'an 710061, P.R. China

E-mail: xaslp@126.com

Key words: renal cell carcinoma, metastasis, parotid region, therapy development the best of our knowledge, this case is the first documented example of such large metastasis of RCC.

The study was approved by the Ethics Committee of The First Affiliated Hospital of Medical College of Xi'an Jiaotong University, Shaan'xi, Xi'an, China, and written informed consent was obtained from the patient.

\section{Case report}

A 44-year-old female visited our hospital due to the presence of a painless mass in her parotid region. The patient denied all genitourinary symptoms and was otherwise well. Laboratory studies revealed normal hemogram, urinalysis and blood ureanitrogen results. The results of a complete ear, nose and throat examination were also normal. The head and neck computed tomography (CT) scan revealed a $4 \times 2.5-\mathrm{cm}$ mass in the right parotid region accompanied by an osteolytic destruction of the right mandibular branch (Fig. 1A). A roentgenogram of the chest was observed to be normal. Further examination by an abdominal CT scan revealed a 6.5-cm mass in the left kidney, suggesting RCC (Fig. 2), and a mass in the right iliac area accompanied by a slightly osteolytic destruction of the right iliac bone (Fig. 3A). A radical nephrectomy was performed. The histological analysis revealed a renal clear cell carcinoma (Fig. 4) and confirmed the renal tumor to be the primary neoplasm. The patient was then discharged from the hospital, as she did not accept any further treatment.

After seven months, the patient presented with the mass in the right parotid region that had enlarged to a ball-like size and was causing a difficulty in opening the mouth. On physical examination, positive findings consisted of a firm mass measuring $\sim 12 \times 13 \mathrm{~cm}$ in the parotid region (Fig. 5). The mass demonstrated no tenderness but had local heat. The CT scan revealed that the masses in the right parotid region and the right iliac area had enlarged to 12.5 and $13.5 \mathrm{~cm}$, respectively, and were accompanied by serious osteolytic destruction of the right mandibular branch and the right iliac bone, respectively (Figs. 1B and 3B). Furthermore, liver metastasis, multiple bone metastases and bilateral multiple metastatic lesions in the lungs were identified. The patient was administered interleukin (IL)-2 and bisphosphonates, 
A

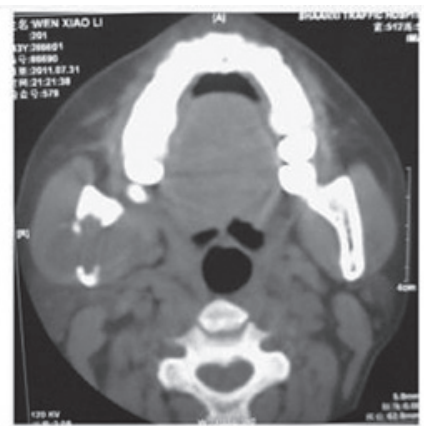

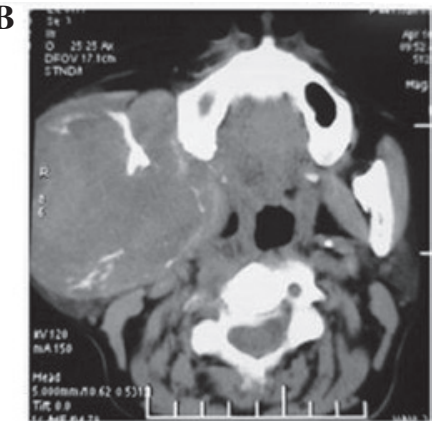

Figure 1. Computed tomography (CT) scan of the head and neck revealing a mass in the right parotid region. (A) Seven months previously: The mass is $\sim 4 \times 2.5 \mathrm{~cm}$ and is accompanied by a slightly osteolytic destruction of the right mandibular branch. (B) At present: The enlarged mass is $\sim 12.5 \mathrm{~cm}$ and is accompanied by serious osteolytic destruction of the right mandibular branch.

A

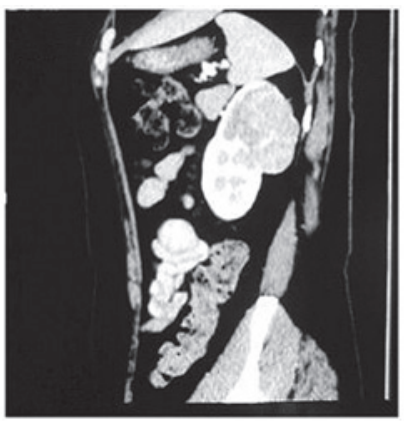

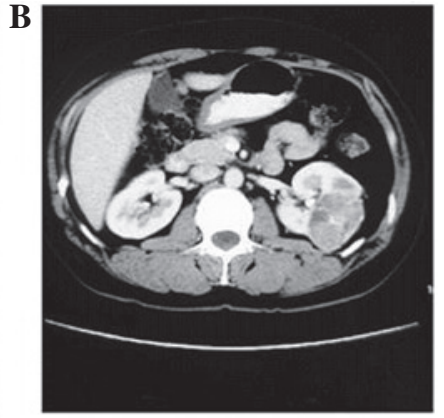

Figure 2. (A) Sagittal and (B) coronal computed tomography (CT) scan demonstrating the mass of the left kidney.

A

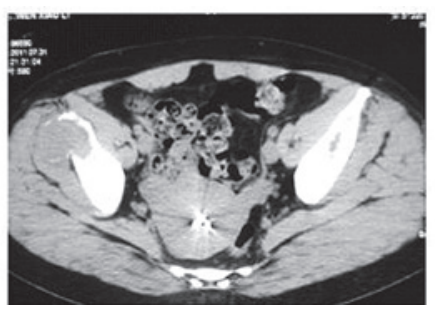

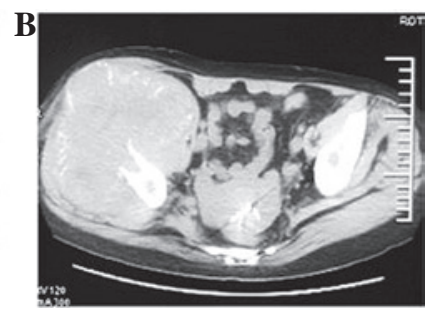

Figure 3. Computed tomography (CT) scan of the pelvic cavity revealing a mass in the right iliac area. (A) Seven months previously: The mass is $\sim 3$ cm and is accompanied with a slightly osteolytic destruction of the right iliac bone. (B) At present: The enlarged mass is $\sim 13.5 \mathrm{~cm}$ and is accompanied with a serious osteolytic destruction of the right iliac bone.

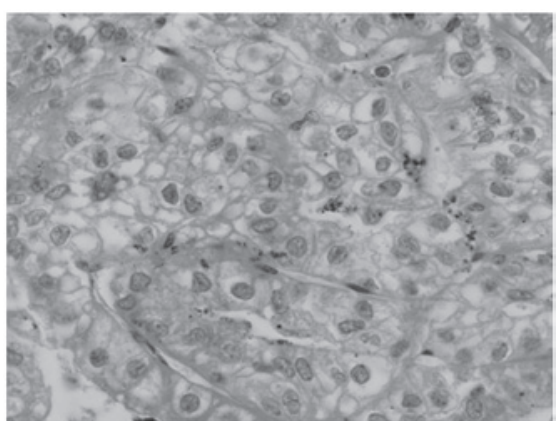

Figure 4. Histological analysis revealed a typical morphology of a middlegrade (grade 2) clear-cell renal cell carcinoma, which confirmed the patient's tumor to be a primary neoplasm. Hematoxylin and esosin (H\&E); magnification, $\mathrm{x} 1,000$.

and received local radiotherapy to the right parotid region and pelvic cavity. However, after three weeks, oral mucositis
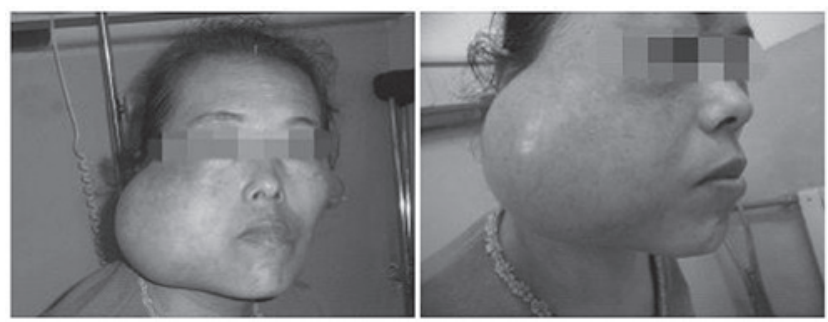

Figure 5. Ball-like mass in the right parotid region.

occurred. Due to unbearable pain, the patient did not accept further radiotherapy. Therefore, targeted therapy with sunitinib was recommended; however, the patient refused this treatment due to its high cost. The patient was treated with Chinese medicine and supportive care. Further follow-up continues. 


\section{Discussion}

In adults, RCC constitutes 2-3\% of all malignancies, accounting for $\sim 90 \%$ of all primary renal tumors, with an incidence peak in the sixth decade of life $(4,5)$. RCC are hypervascular tumors with a high expression of vascular endothelial growth factor (VEGF), the VEGF receptor (VEGFR), the platelet-derived growth factor (PDGF) receptor and basic fibroblast growth factor (bFGF) (6). Cancer cells are also known to have a good adaptive potential in a diverse array of microenvironments, giving rise to the high metastatic potential of RCC (7). Additionally, as the kidney receives $\sim 25 \%$ of the circulating blood volume per minute, the main mechanism of systemic metastases of RCC is hematogenous metastasis. The most common sites for RCC metastasis are the lungs (45.2\%) and bone $(29.5 \%)$, followed by the lymph nodes $(21.8 \%)$ and the liver $(20.3 \%)$ (8). Metastasis to the head and neck region are less common, comprising 14-16\% of all cases (9). The current patient who initially presented with metastasis to the parotid region, which then developed into systemic multiple metastases, was a rare case.

Throughout the past decade, improvements in the understanding of the molecular pathways implicated in the pathogenesis of RCC has led to a marked expansion in the treatment options available to patients with metastatic RCC (mRCC). Previously, systemic treatment was limited to cytokine therapy with IL-2 or interferon (IFN), as mRCC is largely resistant to chemotherapy (10). However, in patients with mRCC, cytokine therapy is correlated with low response and high toxicity rates (10). In recent years, identification of VEGF, VEGFR and the mammalian target of rapamycin (mTOR) as dysregulated signaling pathways in the development and progression of RCC has led to the rapid development of novel molecular targeted therapies. Thus far, six targeted therapies, including sorafenib, sunitinib, bevacizumab (in combination with IFN- $\gamma$ ), temsirolimus, everolimus and pazopanib, have been evaluated in randomized, controlled phase III clinical trials of patients with mRCC and approved by the US Food and Drug Administration (FDA) for the management of mRCC (11). Each of these new agents has demonstrated a significant clinical benefit and fewer detrimental side-effects, leading to a better quality of life for patients (11-17). New targeted agents with novel mechanisms of action are also being studied, including histone deacetylase inhibitors, angiopoietin/tyrosine protein kinase receptor (TIE-2) inhibitors and carbonic anhydrase IX inhibitors (11).

Recent developments have raised the question of whether patients benefit most from combinatorial or sequential therapy of targeted agents. At present, sequential therapy with targeted agents is the standard of care (18). Combinatorial therapy strategies have not yet been demonstrated to be beneficial, with a number of combinations exhibiting excessive toxicity with marginal or inferior efficacy compared with that observed with the sequential use of agents (19). Ongoing investigations concerning the biomarkers that predict severe adverse events and the response of an individual patient to different targeted therapies will lead to a more personalized approach to treating $\operatorname{mRCC}(20,21)$. Additionally, novel immunological therapies are currently being developed to treat mRCC, including those that block cytotoxic
T-lymphocyte antigen 4 (CTLA4) (11). Despite the recent progress, $\mathrm{mRCC}$ remains a disease with no curative therapy and further research is required.

\section{References}

1. Vara A, Madrigal B, Pérez del Río MJ, et al: Parotid metastasis from renal clear cell adenocarcinoma. An unusual site for metastasis. Urol Int 61: 196-198, 1998.

2. Pisani P, Angeli G, Krangli M and Pia F: Renal carcinoma metastasis to the parotid gland. J Laryngol Otol 104: 352-354, 1990.

3. Leibovich BC, Pantuck AJ, Bui MH, et al: Current staging of renal cell carcinoma. Urol Clin North Am 30: 481-497, 2003.

4. Karumanchi SA, Merchan J and Sukhatme VP: Renal cancer: molecular mechanisms and newer therapeutic options. Curr Opin Nephrol Hypertens 11: 37-42, 2002.

5. Suh JH, Oak T, Ro JY, et al: Clinicopathologic features of renal cell carcinoma in young adults: a comparison study with renalcell carcinoma in older patients. Int J Clin Exp Pathol 2: 489-493, 2009.

6. Mena AC, Pulido EG and Guillén-Ponce C: Understanding the molecular-based mechanism of action of the tyrosine kinase inhibitor: Sunitinib. Anticancer Drugs 21 (Suppl 1): S3-S11, 2010.

7. Miah MS, White SJ, Oommen G, et al: Late simultaneous metastasis of renal cell carcinoma to the submandibular and thyroid gland seven years after radical nephrectomy. Int J Otolaryngol 2010: 698014, 2010.

8. Bianchi M, Sun M, Jeldres C, et al: Distribution of metastatic sites in renal cell carcinoma: a population-based analysis. Ann Oncol. Ann Oncol 23: 973-980, 2012.

9. Sarkis P, Bou-Malhab F and Mouaccadieh L: Solitary laryngeal metastasis from clear cell carcinoma of the kidney: Clinical case and review of the literature. Prog Urol 22: 307-309, 2012 (In French).

10. Oudard S, George D, Medioni J and Motzer R: Treatment options in renal cell carcinoma: past, present and future. Ann Oncol 18 (Supl 10): x25-x31, 2007.

11. Matrana MR, Atkinson B, Jonasch E and Tannir NM: Emerging targeted therapies in metastatic renal cell carcinoma. Curr Clin Pharmacol 6: 189-198, 2011.

12. Motzer RJ, Hutson TE, Tomczak P, et al: Overall survival and updated results for sunitinib compared with interferon alfa in patients withmetastatic renal cell carcinoma. J Clin Oncol 27: 3584-3590, 2009.

13. Escudier B, Bellmunt J, Négrier S, et al: Final results of the phase III, randomized, double-blind AVOREN trial of first-line bevacizumab (BEV) plus interferon-alpha $2 \mathrm{a}$ (IFN) in metastatic renal cell carcinoma (mRCC). J Clin Oncol (Meeting Abstracts) 27 (Suppl): 15S, 2009.

14. Sternberg CN, Davis ID, Mardiak J, et al: Pazopanib in locally advanced or metastatic renal cell carcinoma: results of a randomized phase III trial. J Clin Oncol 28: 1061-1068, 2010.

15. Hudes G, Carducci M, Tomczak P, et al; Global ARCC Trial: Temsirolimus, interferon alfa, or both for advanced renal-cell carcinoma. N Engl J Med 356: 2271-2281, 2007.

16. Escudier B: Sorafenib for the management of advanced renal cell carcinoma. Expert Rev Anticancer Ther 11: 825-836, 2011.

17. Motzer RJ, Escudier B, Oudard S, et al; RECORD $\square 1$ Study Group: Phase 3 trial of everolimus for metastatic renal cell carcinoma: Final results and analysis of prognostic factors. Cancer 116: 4256-4265, 2010.

18. National Comprehensive Cancer Network. NCCN Clinical Practice Guide-lines in Oncology ${ }^{\mathrm{TM}}$. Kidney Cancer. V.2.2010. Available at http://www.nccn.org/professionals/physician_gls/ PDF/kidney.pdf, accessed March 26, 2010.

19. Hutson TE: Targeted therapies for the treatment of metastatic renal cell carcinoma: clinical evidence. Oncologist 16 (Suppl 2): 14-22, 2011.

20. Mizuno R and Oya M: Biomarkers of response to molecular targeted therapy in renal cell carcinoma. Gan To Kagaku Ryoho 38: 1088-1091, 2011 (In Japanese).

21. Eisengart LJ, MacVicar GR and Yang XJ: Predictors of response to targeted therapy in renal cell carcinoma. Arch Pathol Lab Med 136: 490-495, 2012. 